\title{
POST-MEDICINA: UNA ALTERNATIVA ECOPOLITICA AL PARADIGMA BIOMÉDICO
}

\section{POST-MEDICINE: AN ECOPOLITICAL ALTERNATIVE TO THE BIOMEDICAL PARADIGM}

\author{
Abel Novoa. Universidad de Murcia \\ <abelnovoajurado@gmail.com>
}

Resumen: El aumento imparable del gasto público sanitario cuestiona la vigencia de las políticas sanitarias de los países con Estados del Bienestar. Este artículo revisa las contribuciones científicas de autores que comparten el enfoque innovador de la post-medicina; alternativo al paradigma bio-medico, en crisis. Tales aportaciones al conocimiento y a la resolución del problema van más allá de polemizar sobre los recortes de la administración sanitaria. Los sistemas sanitarios públicos muestran debilidades estructurales claves, derivadas de: un "derecho a la salud" transformado en "deseo a no sufrir"; olvido de otras políticas importantes en la calidad de vida y salud; y los efectos mórbidos del Complejo Corporativo Consumista (CCC) (Freudemberg, 2014). En la discusión de propuestas de mejora, el autor aplica la perspectiva del decrecimiento. Concluye estableciendo una agenda para aumentar la sostenibilidad interna y externa; evitar la deriva institucional y las malas prácticas corporativas; así como implicar a los ciudadanos como agentes colectivos del cambio.

Palabras clave: post-medicina, burbuja sanitaria, gasto público, salud, Complejo-Corporativo-Consumista, decrecimiento, sistema nacional de salud, paradigma biomédico, medicina.
Summary: The unstoppable increase in public health spending brings into question the viability of health policies in welfare state countries. This article reviews the scientific contributions of authors who agree with the innovative postmedicine approach as opposed to the bio-medical paradigm. Such contributions to knowledge and problem solving go beyond arguing about budget cuts in health administration. Public health systems show key structural weaknesses, namely: a shift from the "right to health" towards "a desire to not suffer"; ignoring of policies important for quality of life and health; and deadly effects of the Corporate-Consumption Complex (CCC) (Freudemberg, 2014). In discussing proposals for improvement, the author applies a degrowth perspective. He recommends setting an agenda that increases internal and external sustainability, avoids institutional drift and corporate wrongdoing, and involves citizens as agents of change.

Key words: post-medicine, health bubble, public expenditure, health, Corporate-Consumption Complex, degrowth, national health system, biomedical paradigm, medicine 


\section{Introducción}

En los países con más de medio siglo de sanidad pública, el gasto público en políticas sanitarias por habitante ha sido cada vez más elevado y, sin embargo su rentabilidad social ha sido decreciente. Desde el discurso continuista de la "bio-medicina", las propuestas al respecto se orientan a recortar gastos y privatizar gestión y servicios. Por otra parte, el conjunto de autores que agrupamos en la post-medicina (Novoa, 2013) ofrecen una interpretación estructural de la crisis de la sanidad pública, profundizan en los aspectos estructurales involucrados en revertir el proceso y aportan propuestas relativas a las políticas y a la ciudadanía.

El objetivo del presente artículo es investigar el estado actual de las aportaciones de la post-medicina, como aportaciones alternativas al paradigma biomédico dominante, en la interpretación y resolución de la crisis de la sanidad pública. La metodología empírica y teórica seguida ha consistido en recoger, analizar y sintetizar aportaciones significativas del debate entre biomedicina y post-medicina.

La postmedicina agrupa un conjunto de pensadores y practicantes mayormente procedentes del ejercicio de la medicina y de la política sanitaria con publicaciones en revistas científicas sobre el tema desde 2010 a 2015. Un momento álgido de la polémica internacional se produjo con la publicación del libro de N.Freudenmberg (2014) "Letal pero legal".

Entre los contribuyentes a la post-medicina cabe distinguir entre autores que han reflexionado sobre las cuestiones que enmarcan el problema o vislumbran las tendencias, respecto de los autores que profundizan en aspectos específicos.

Dentro del primer grupo son especialmente destacables diversas aportaciones. D. Callahan que, entre 1997 a 2000, publica varios trabajos sobre una medicina sostenible y los límites médicos en sociedades que envejecen; se publicó en español en 2004. Otras aportaciones clave son las de los autores JR Repullo y A.Segura (2006) sobre la sostenibilidad de la sanidad pública. Estos autores, en términos estrictamente económicos, critican la ineficiencia agregada de todo el sistema biomédico en el que un gasto imparable e implacable habría dejado de corresponderse con una mejora proporcional de la salud, la calidad de vida de los ciudadanos y la equidad social (Repullo y Segura, 2006). Otros trabajos que aportan a la comprensión de la problemática son los de la Organización Mundial de la Salud (OMS) de los años 2008 y 2009. Sus informes apuntan a: una polarización del gasto hacia la atención hospitalaria, los medicamentos y las tecnologías en detrimento de la atención primaria y la salud pública (OMS, 2008); y a un continuo proceso de supresión de cuestiones fundamentales como la importancia de los determinantes sociales sobre la atención sanitaria en la mejora de la salud (OMS, 2009).

Y por último, como análisis marco incluimos en este grupo a la aplicación a la sanidad de las aportaciones de S.Latouche (2008) sobre el "decrecimiento (Novoa, 2013b). Así alinea la crisis de la sanidad en un contexto más global, aplicando el enfoque del decrecimiento a la sanidad, en el sentido de "decrecer en sanidad para crecer en salud y equidad" (Novoa, 2013b).

En cuanto a los autores de la postmedicina aportan análisis sobre cuestiones específicas de la crisis de la sanidad, destacan igualmente diversas aportaciones: los análisis de rendimientos decrecientes de la inversión pública en sanidad (Fuchs, 2004); el sobre-diagnóstico, la sobre-medicación (Gérvas, Fernández, 2014) y la medicalización de la vida (Gérvas, Fernández, 2012); los crecientes problemas de seguridad 
de los pacientes (Kohn, Corrigan, Donaldson, 2000); la excesiva variabilidad de la práctica clínica (Peiró, Meneu, Bernal, 2005); la influencia indebida de la industria farmacéutica y tecnológica sobre la investigación médica, los sistemas de salud y la práctica clínica (Stamatakis, Weiler, loannidis, 2013; la influencia de las farmacéuticas en el sistema de salud Gøtzche, 2014);

La síntesis y análisis de las aportaciones se ha estructurado en tres partes, a continuación. En la primera se debate la transformación del concepto de salud como derecho a salud como "no sufrimiento" que destacan los pensadores de la post-medicina. En la segunda parte de aborda el significado del término "Complejo Corporativo Consumista". Y en la tercera y cuarta parte se recoge el resultado del análisis de las propuestas de la post-medicina en los ámbitos de la política y de la ciudadanía, respectivamente.

\section{La crisis de la sanidad pública desde la post-medicina}

La crisis sanitaria se enmarca en un entorno estructural de crisis financiera mundial, unida a la crisis medioambiental (cambio climático, agotamiento de los combustibles fósiles, incremento de la huella ecológica) y a la crisis social (aumento de las desigualdades y de la pobreza tanto dentro de las sociedades desarrolladas como entre el Norte y el Sur del planeta) (Novoa, 2013b). La propia crisis estructural pondría de manifiesto la imposibilidad de seguir alimentando un modelo basado en el crecimiento económico infinito, el progreso tecnológico dirigido por el mercado y el hiper-consumo.

El decrecimiento económico (Latouche, 2008; Taibo, 2009) es el paradigma que emerge con más capacidad para aportar un enfoque, a la vez humanista y ecológicamente sostenible, a los retos de la "ciclogénesis explosiva" en la que nos encontramos. Su aplicación a la atención sanitaria y los servicios de salud está en elaboración. Desde una perspectiva postmédica se trataría de "decrecer en atención sanitaria para crecer en salud y equidad" como enfoque alternativo frente a soluciones que tan solo propugnan "mejor gestión", en un sistema de salud que ha perdido la visión de sus fines (Hasting, 1996). El paradigma decrecentista aplicado a la medicina y a la atención sanitaria implica un cambio de mentalidades, prioridades y conceptos.

El sistema público de salud se ha convertido en un fin en si mismo olvidando sus objetivos sociales primordiales. Es una especie de "agujero negro" presupuestario que ha impuesto su jerarquía gracias a las demandas sociales, el prestigio de "lo científico" y los réditos electorales.

En EE.UU se ha calculado que entre el 21 y el $47 \%$ de todo el presupuesto sanitario del año 2011 se malgastó; la cuarta parte de esta ineficiencia correspondía a intervenciones, tecnologías o medicamentos inútiles o innecesarios; el resto, a fallos en la atención y coordinación, mala gestión, alteración de los precios y fraudes (Berwick, Andrew, Hackbarth, 2012).

Además, los presupuesto dedicados a salud impiden la financiación adecuada de otras políticas con mayor capacidad de generar salud, como las sociales, educativas, de vivienda, empleo o medio-ambientales (OMS, 2009). Donald Berwick ha denominado a este proceso, "confiscación" de la riqueza de los países por parte de los presupuesto sanitarios (Berwick, 2014). 


\subsection{El individualismo cambia el concepto de salud}

Esta "burbuja sanitaria" tiene múltiples razones: culturales, profesionales, científicas, históricas, etc. Repullo y Segura (2006) señalan, por ejemplo, el conflicto histórico latente que siempre ha existido entre una perspectiva clínica centrada en el beneficio individual y otra más holística. Esta última, según los citados autores, percibió las "limitaciones de la clínica frente a las dimensiones sociales de los problemas de salud" y propuso en su día priorizar acciones de índole política y comunitaria sobre los enfoques más individualistas.

Tras diversos intentos infructuosos de acomodar ambas visiones -el más importante, la Conferencia de Alma Ata de 1978 que planteó la atención primaria y comunitaria como base de los sistemas de salud- el cambio político neoliberal de los años 80 hizo emerger, para quedarse, la centralidad política y económica del individuo. En palabras de Repullo y Segura (2006) se impuso el "ethos del mercado":

"Posiblemente esta cura de individualidad ha tenido algunos aspectos positivos, como respuesta a sociedades más maduras, que no querían seguir siendo pacientes o súbditos pasivos de la medicina o del Estado, pero al coste de que el ethos del mercado anidara sin complejos en la sociedad, en el Estado y en la medicina" (Repullo y Segura, 2006)

La hegemonía de los valores individualistas y su correlato económico, el mercado y el consumo, ha tenido importantes consecuencias en medicina. Sin ir más lejos, el propio concepto de salud ha adquirido una significación desproporcionada en un inconsciente colectivo dominado por la satisfacción de los deseos. A este respecto, destacamos las palabras del bioeticista norteamericano Daniel Callahan:

"pelear contra la enfermedad, el envejecimiento y la muerte es la idea (o al menos una de las ideas) que da sentido al ser humano...; la salud se ha convertido en un fin en sí mismo y la lucha contra el sufrimiento, en una fuente de significado" (Daniel Callahan, 1998:31).

Tabla 1: Fines tácitos del paradigma de la medicina científica

\begin{tabular}{|l|}
\hline Curar ignorando el cuidar \\
\hline Aumento indefinido de la expectativa de vida \\
\hline Alivio de todo sufrimiento mental y físico que la medicina científica sea capaz de controlar \\
\hline Satisfacción de todos los deseos en salud que puedan ser colmados mediante medios técnicos \\
\hline $\begin{array}{l}\text { Permitir que sean el progreso médico y la innovación tecnológica los responsables de redefinir } \\
\text { constantemente los fines de la atención sanitaria y el ámbito de la salud }\end{array}$ \\
\hline
\end{tabular}

Fuente: elaboración propia a partir de Callahan, 2000.

En la tabla 1 resumimos lo que Daniel Callahan (2000) viene a considerar los fines tácitos como son que se priorice la curación frente al cuidado y se apoyarán en la medicina técnica y en las tecnologías como prioridad de la atención sanitaria. Esta búsqueda sublimada, casi religiosa, de la salud ha sido fácilmente instrumentalizada por el mercado y su perturbador Caballo de Troya: la innovación tecnológica:

La combinación de factores sociales (como el individualismo o la pulsión contemporánea por la búsqueda de una mejor salud como fin en si misma), científicos (el progreso tecnológico guiado por el mercado y la transformación paulatina de la tec- 
nología médica, los medicamentos y las intervenciones sanitarias en productos de consumo convenientemente publicitados), profesionales (una educación médica cada vez más centrada en las tecnologías y medicamentos; una generalizada colusión de intereses profesionales, académicos y científicos con el poder económico de las grandes corporaciones) y políticos (la atención sanitaria centrada en los grandes hospitales y tecnologías como instrumento para ganar votos, en detrimento de la atención primaria o la salud pública) ha sido un cóctel que ha derivado en una irracionalidad financiera sin fondo y un proyecto, el de la biomedicina, que ha perdido el sentido, dirigido por fines tácitos irracionales (Tabla 1) que producen graves consecuencias sociales. (Tabla 2 ).

En este proceso, señala D.Callahan (1987) reflexiona sobre la tendencia a que el listón de lo que se considera buena salud o de las expectativas sobre la vejez, hayan ido subiendo a la para que su presión sobre el sistema sanitario:

“¿Qué es lo que consideramos buena salud o duración adecuada de la vida?. Estas cuestiones son cada vez más difíciles de responder, principalmente debido al hecho de que el progreso médico hace avanzar constantemente las fronteras de la salud e invita, incluso seduce, a establecer un estándar cada vez más elevado de lo que debería considerarse como buena salud y una duración aceptable de la vida" (Callahan, 1987: pag. 25)

Tabla 2: Las consecuencias del paradigma biomédico

\begin{tabular}{|l|l|}
\hline Consecuencia & Procesos sociales, culturales y tecnológicos implicados \\
\hline $\begin{array}{l}\text { Insostenibilidad } \\
\text { económica de los } \\
\text { sistemas de salud }\end{array}$ & $\begin{array}{l}\text { Debido a la combinación de envejecimiento de la sociedad, desarrollo } \\
\text { tecnológico e intensificación de su uso, y demandas crecientes de la } \\
\text { población estimuladas por el mercado y los modelos sociales. }\end{array}$ \\
\hline \multirow{2}{*}{$\begin{array}{l}\text { La tiranía de la } \\
\text { salud, el riesgo y el } \\
\text { perfeccionamiento }\end{array}$} & $\begin{array}{l}\text { Tiranía de la salud: Creencia generalizada de que solo se puede } \\
\text { conseguir buena salud si la medicina sigue mejorando y los sistemas } \\
\text { sanitarios obteniendo más financiación. }\end{array}$ \\
\cline { 2 - 3 } & \begin{tabular}{l} 
Tiranía del riesgo: Convencimiento por parte de la población de que \\
cualquier enfermedad o dolencia está causada por un accidente bioló- \\
gico que siempre puede ser evitado mediante mejor y más prevención, \\
\cline { 2 - 3 } mejón sanitaria y más conocimiento científico.
\end{tabular} \\
\cline { 2 - 2 } & $\begin{array}{l}\text { Tiranía del perfeccionamiento: La certeza por parte de los ciudadanos } \\
\text { de que es posible eliminar todos los riesgos para la salud y todas las } \\
\text { causas conocidas de enfermedad reduciendo a cero la posibilidad de } \\
\text { muerte o daño que existen. }\end{array}$ \\
\hline \multirow{2}{*}{$\begin{array}{l}\text { Confusión acerca } \\
\text { del campo propio de } \\
\text { la medicina }\end{array}$} & $\begin{array}{l}\text { El desarrollo científico y las demandas de la población hace que la } \\
\text { atención médica esté cada vez más orientada hacía la satisfacción de } \\
\text { deseos individuales (como los estéticos o psicológicos) o la solución de } \\
\text { problemas sociales (como la violencia o el abuso de sustancias) que } \\
\text { poco tienen que ver con los fines de la medicina. }\end{array}$ \\
\hline $\begin{array}{l}\text { El sesgo en las } \\
\text { prioridades }\end{array}$ & $\begin{array}{l}\text { La priorización de la medicina hacia la curación de enfermedades y la } \\
\text { extensión de la vida ha producido un olvido del cuidado y la paliación. }\end{array}$ \\
\hline $\begin{array}{l}\text { El incremento de las } \\
\text { desigualdades entre } \\
\text { naciones y clases } \\
\text { sociales }\end{array}$ & $\begin{array}{l}\text { Debido a la atracción y capacidad de seducción del avance científico se } \\
\text { produce un incremento desproporcionado de la atención hospitalaria, } \\
\text { más cara y con menos capacidad de disminuir las desigualdades en sa- } \\
\text { lud, en detrimento de la atención primaria y las políticas de salud pública. }\end{array}$ \\
\hline
\end{tabular}

Fuente: elaboración propia a partir de Callahan, 1998, p 74-79. 


\subsection{Paradoja: la ciudadanía alejada del debate}

Como sintetizamos en la tabla 2 y se desprende del análisis de Callahan (1998) las expectativas generadas entre la población, sobre salud, enfermedad y vejez, han adoptado la forma de "tiranías" y en presión social hacia el gasto ilimitado en sanidad.

D. Callahan (1998) diferencia tres formas de tiranía, la de la salud, la del riesgo y la del perfeccionamiento. La tiranía de la salud se resume en la creencia de que sólo mejora la salud gastando mas en medicina y en sanidad. La tiranía del riesgo consistente en la creencia de que toda enfermedad se corresponde con un accidente biológico que puede ser evitado gastando mas en atención, prevención e investigación. Y la tiranía del perfeccionamiento que se basa en la creencia de que se pueden llegar a reducir a 0 los riesgos y las enfermedades.

Cuando en los últimos dos años se anunciaron en España, sin grandes preámbulos ni explicaciones, limitaciones presupuestarias para el gasto sanitario, las movilizaciones ciudadanas de oposición a los "recortes" estuvieron justificadas en una necesaria resistencia civil ante reformas ideológicamente dirigidas. Sin embargo, en los foros ciudadanos se obvia el debate de la postmedicina tendiéndose mas hacia un discurso de "resistencia a los recortes" enfrascado en volver al paradigma sanitario del estado del bienestar de atención médica ilimitada y profundamente contradictoria. Tal contradicción radicaría en que su financiación pública responde a fines intrínsecamente individualistas, irracionales, y supone un enorme esfuerzo financiero que propugna intervenciones sanitarias cada vez más alejadas de cualquier sentido socialmente productivo.

La equiparación del derecho a la salud con la exigencia social a no tener sufrimiento, enfermedades o riesgos (Callahan, 1998:75) hace que se confundan las prioridades y que una población legítimamente indignada sintonice con las corporaciones multinacionales que ven una excelente oportunidad para proteger sus intereses comerciales -iqué paradójico!- en la defensa política de un sistema público de salud que no ha sido capaz de separar, "el trigo de la paja", es decir, lo eficaz (beneficio de una intervención, medicamento o tecnología en condiciones ideales o experimentales) de lo efectivo (beneficio en condiciones reales o usuales).

¿Por qué la sociedad muestra una sensibilidad tan exquisita ante, por ejemplo, el despilfarro de los ayuntamientos en obras y servicios (polideportivos, centros de congresos, etc..) y es tan escasa ante el que se produce, en mayor magnitud, en el sistema de salud?

Los profesionales sanitarios ante la crisis de financiación han optado -con espléndidas excepciones como la "marea blanca" en Madrid- por un "repliegue corporativista" y asisten, casi como espectadores, al desmantelamiento del Sistema Nacional de Salud. Ante la ausencia de un debate sobre el problema estructural, los profesionales sanitarios tienden a encontrar solamente justificación para alzar su voz cuando se ven atacados sus propios intereses gremiales (Novoa, 2013). Este artículo precisamente pretende contribuir a redimensionar el debate considerando que la polémica "recortes si, recortes no" resulta reducida a la vista de la complejidad de los cambios que se avecinan en el sistema médico y las oportunidades de los profesionales de expresarse y posicionarse al respecto.

Los autores de la postmedicina, además de alertar sobre las expectativas erráticas en salud que se han generado en la ciudadanía y que tiranizan los sistemas sanitarios de los estados de bienestar, también aportan análisis sobre las oportunidades de reducción del despilfarro. 
Ya hay algunos datos significativos que expresan las oportunidades de reducción del despilfarro. Así, sólo empleando el equivalente terapéutico más barato en dos grupos de medicamentos muy utilizados como los inhibidores de la bomba de protones (IBPs) y los empleados en disminuir el colesterol, en el año 2010, se hubieran ahorrado casi 1000 millones de euros en toda España. La reducción de la prescripción inadecuada de ambos fármacos, superior a la tercera parte del total, se estima que todavía incrementaría más las cifras de ahorro (Peiró, 2012).

En la Comunidad Autónoma de la Región de Murcia, según cálculos propios, el ahorro potencial con esta medida, solo en un mes, es semejante al presupuesto de la CCAA dirigido a financiar las rentas mínimas de inserción de todo un año. Las partidas dedicadas a sanidad se llevan entre el 35 y el $45 \%$ de los presupuestos reales de las CCAA (Peiró, 2012), impidiendo la financiación de otras políticas públicas igual o más importantes en la generación de salud, y con más capacidad para mejorar la calidad de vida de los ciudadanos y la equidad social (Cortés-Franch y González, 2014).

Así, la sanidad pública se ha convertido en un gigantesco mercado subvencionado. Entre las consecuencias indeseables destacan: (1)que el gasto sanitario creciente absorbe financiación pública que podría destinarse a otras políticas con mayor capacidad de generar salud y equidad, como la educación para la salud o y políticas de empleo; (2) que alrededor de ese gasto público se organiza un complejo corporativo consumista sanitario (CCCS) a modo de red de compañías que actúan como oligopolios mundiales (Freundemberg,2014).

\section{El papel del Complejo Corporativo Consumista Sanitario (CCCS)}

Además de las medidas de reducción del despilfarro sanitario, otro ámbito de intervención que destacado por autores de la postmedicina consiste en la expansión de morbilidad generada por el Complejo Corporativo Consumista Sanitario (en adelante CCCS).

Según indica Freudemberg (2014), el CCCS está compuesto por una red de compañías (alimentación, automóviles, farmacéuticas, tabaco, alcohol y armas) que actúan en régimen de oligopolios mundiales. Junto con instituciones financieras, académicas, científicas, profesionales, aseguradoras privadas y empresas de comunicación y publicidad, y con el único objetivo de obtener beneficios económicos, han impuesto un patrón de hiper-consumo de productos no saludables, que es la principal causa de la emergencia de las enfermedades no transmisibles (también llamadas crónicas) como las cardiovasculares, cáncer, respiratorias y diabetes (Freudemberg, 2014).

Los papeles se reparten: los modelos de negocio de las empresas alimentarias, alcohol, tabaco, armas y automóviles generan la morbilidad; las instituciones académicas y profesionales, dominadas por la agenda de la industria farmacéutica, justifican "científicamente" la sobre-actuación médica que, convenientemente publicitada, genera un hiperconsumo de productos farmacéuticos, tecnologías sanitarias e intervenciones médicas que, a su vez, contribuyen netamente a la expansión de la morbilidad. Gøtzsche (2014) ha aportado suficientes datos como para poder afirmar que en EE.UU y la UE "Ios medicamentos son la tercera causa de muerte después de las cardiopatías y el cáncer” (Gøtzsche, 2014: 378)). 
Tabla 3: Muertes anuales atribuibles directa o indirectamente al modelo de negocio basado en el hiper-consumo

\begin{tabular}{|c|c|c|c|}
\hline $\begin{array}{l}\text { Clase de } \\
\text { Industria } \\
\text { implicada }\end{array}$ & $\begin{array}{l}\text { Principales problemas de } \\
\text { salud relacionados }\end{array}$ & $\begin{array}{l}\text { Muertes anua- } \\
\text { les estimadas } \\
\text { en USA }\end{array}$ & $\begin{array}{l}\text { Muertes anuales estima- } \\
\text { das en todo el mundo }\end{array}$ \\
\hline Tabaco & $\begin{array}{l}\text { Enfermedades cardiovas- } \\
\text { culares, cáncer de pulmón } \\
\text { y otros, enfermedades } \\
\text { respiratorias }\end{array}$ & 467.000 & $\begin{array}{l}6.297 .287 \text { (incluye muertes } \\
\text { de fumadores pasivos) }\end{array}$ \\
\hline $\begin{array}{l}\text { Alimentación y } \\
\text { bebidas }\end{array}$ & $\begin{array}{l}\text { Obesidad, diabetes, enfer- } \\
\text { medades cardiovasculares, } \\
\text { algunos cánceres }\end{array}$ & $\begin{array}{l}216.000 \\
\text { (atribuidas a la } \\
\text { obesidad) }\end{array}$ & $\begin{array}{l}3.371 .232 \text { (atribuidas a la } \\
\text { obesidad) }\end{array}$ \\
\hline Alcohol & $\begin{array}{l}\text { Accidentes, homicidios, } \\
\text { cáncer, cirrosis }\end{array}$ & 64.000 & 4.860 .168 \\
\hline $\begin{array}{l}\text { Vehículos de } \\
\text { motor }\end{array}$ & $\begin{array}{l}\text { Accidentes, enfermedades } \\
\text { debidas a la contamina- } \\
\text { ción (respiratorias, cáncer, } \\
\text { cardiovasculares) }\end{array}$ & 43.667 & $\begin{array}{l}1.300 .000 \text { (por acci- } \\
\text { dentes); } 3.223 .540 \text { (por } \\
\text { contaminación) }\end{array}$ \\
\hline Armas de fuego & $\begin{array}{l}\text { Homicidios, suicidios y } \\
\text { accidentes }\end{array}$ & 30.694 & $\begin{array}{l}300.000 \text { en conflictos béli- } \\
\text { cos; } 200.000 \text { por homici- } \\
\text { dios, suicidios y accidentes }\end{array}$ \\
\hline Farmacéuticas & Sobre e inframedicación & 128.000 & 200.000 (en Europa) \\
\hline
\end{tabular}

Fuente: adaptado de Freudemberg, 2014

Según se recoge en la Tabla 3, elaborada en base a datos aportados por Freudemberg (2014), las muertes atribuidas al tabaco, el alcohol, la alimentación, etc. se estima que ascienden a unos 15,5 millones al año a nivel mundial. Por lo cual se puede considerar que, con la salvedad de las muertes por envejecimiento, la del CCCS, al ser evitable, se ha convertido en el mayor problema prevenible de salud en el mundo (Freudemberg, 2014) (véaseTabla 3).

Las estrategias de salud pública habrían de cambiar profundamente para enfrentarse a esta nueva amenaza derivada del modelo económico. Podrían aplicarse acciones políticas, fiscalizadoras, regulatorias y también socio-culturales, semejantes a las que se han utilizado en el pasado, por ejemplo, en la lucha contra el fraude fiscal o el cambio climático. Serían politicas que pasarían a un primer plano, dejando en un segundo plano intervenciones que se han mostrado repetidamente insuficientes como la educación para la salud o la prevención (Freudemberg, 2014).

La crisis económica, no obstante, está debilitando gravemente la capacidad de los organismos públicos para defender la salud pública mientras que aumenta el poder de las corporaciones mulnacionales (I.Hernández y B.Lumbreras, 2014) 


\subsection{Daños indirectos del CCCs sobre la salud}

Pero además del daño directo, el CCCS estaría produciendo un daño indirecto grave a través de dos mecanismos. El primero, la degradación del medio ambiente y el cambio climático (Griffiths, 2009), debido a la fuerte dependencia que el CCCS tiene del transporte de mercancías, la agricultura intensiva, la expansión de la ganadería o la utilización en las cadenas de fabricación de combustibles y materiales derivados del petróleo.

El segundo mecanismo tiene que ver con los requerimientos políticos y económicos que necesita el modelo de funcionamiento del CCCS que condiciona una situación creciente de desigualdad e inequidad social que se ha demostrado directamente perjudicial para la salud (Marmot, 2004): cortoplacismo, externalización de riesgos financieros y de daños, desregulación, desgravaciones y baja carga impositiva a las empresas, privatización y concentración oligopólica (Freudemberg, 2014).

Las prácticas del CCCS tienen ciertas características en común: utilizan la ciencia y la tecnología para mejorar sus beneficios (sin reparar en manipulación de las evidencias y fraude científico, estableciendo prioridades de investigación basadas en la previsión de retornos económicos, etc..); diseño y promoción agresiva de productos sin una evaluación adecuada de su impacto en la salud de las personas; realización de esfuerzos deliberados para minimizar las pruebas acerca de los daños que producen sus productos y exageración de sus beneficios.

Otras estrategias que practican consisten en combinar ventas masivas de productos insanos a bajo precio -gracias a prácticas monopolísticas-, con estrategias que impiden el acceso de millones de personas a productos esenciales (es el caso de la industria alimentaria o de la farmacéutica). Por otra parte tenemos prácticas consistentes en transferir o externalizar los efectos adversos de sus productos y prácticas a los consumidores y contribuyentes. Así como promover el consumo de productos dañinos, especialmente entre poblaciones vulnerables como los niños y grupos con adicciones o problemas psico-emocionales (Freudemberg, 2014; Hernández y Lumbreras, 2014).

El daño para la salud de la actividad del CCCS solo se entiende que sea posible porque tambien se ha producido una paulatina degradación democrática en nuestras instituciones. De tal manera que el poder de las corporaciones multinacionales ha dispuesto del terreno libre para obtener el debilitamiento de las regulaciones y los estándares establecidos para proteger la salud pública y el medio ambiente. Hernández y col. (2104) lo denominan "captura de políticas" y lo explican con las siguientes palabras:

"una forma de corrupción, ahora generalizada, mediante la cual grupos de interés controlan áreas políticas dentro de un estado o a nivel supranacional (Unión Europea, Naciones Unidas, etc.), de forma que los gobiernos no pueden, y a menudo no quieren, formular políticas alineadas con el interés general". (Hernández y col., 2014: 25).

La captura de políticas tendría una poderosa base ideológica común que es necesario identificar para poder desactivar (véase Tabla 4) 
Tabla 4: La ideología detrás del modelo de negocio del CCCS y argumentos en contra

\begin{tabular}{|c|c|}
\hline $\begin{array}{l}\text { ARGUMENTO AUTOJUSTIFICATI- } \\
\text { VO DEL CCCS }\end{array}$ & ARGUMENTOS DE LA POSTMEDICINA \\
\hline $\begin{array}{l}\text { Los estilos de vida son los que influ- } \\
\text { yen principalmente en la salud. Los } \\
\text { individuos, son responsables de sus } \\
\text { estilos de vida y comportamientos y } \\
\text { no las compañías o los gobiernos, }\end{array}$ & $\begin{array}{l}\text { Los estilos de vida son, en realidad, condiciones de } \\
\text { vida. Fumar, beber alcohol o estar obeso, por ejemplo, } \\
\text { está directamente relacionado con determinantes } \\
\text { socioeconómicos como el nivel educativo, los ingresos } \\
\text { anuales, la situación laboral o la accesibilidad a zonas } \\
\text { verdes }\end{array}$ \\
\hline $\begin{array}{l}\text { Las compañías producen los produc- } \\
\text { tos que los consumidores quieren. } \\
\text { Si la gente no los demandara, las } \\
\text { compañías no los producirían }\end{array}$ & $\begin{array}{l}\text { La venta masiva de productos insanos está basada en } \\
\text { la publicidad y la demanda inducida a través de la cons- } \\
\text { trucción de necesidades (la invención y exageración de } \\
\text { enfermedades es un buen ejemplo) }\end{array}$ \\
\hline $\begin{array}{l}\text { La educación es la mejor manera } \\
\text { de ayudar a las personas a tomar } \\
\text { buenas decisiones }\end{array}$ & $\begin{array}{l}\text { La educación es condición necesaria pero no suficiente. } \\
\text { La coaptación de la formación médica continuada y la } \\
\text { manipulación de la evidencia científica por la industria } \\
\text { farmacéutica son buen ejemplo de las limitaciones de la } \\
\text { educación. }\end{array}$ \\
\hline $\begin{array}{l}\text { Los gobiernos no deben decirle a las } \\
\text { personas cómo deben vivir. El go- } \\
\text { bierno es el problema, no la solución. }\end{array}$ & $\begin{array}{l}\text { No se trata de entrometerse en las decisiones libres de } \\
\text { los ciudadanos sino de garantizar que esas decisiones } \\
\text { se toman libremente (con información adecuada, sin } \\
\text { coacciones o con estímulos desproporcionados) y se } \\
\text { protege a los colectivos con una autonomía más debili- } \\
\text { tada como los niños o los enfermos }\end{array}$ \\
\hline $\begin{array}{l}\text { Los gobiernos no tienen que decir a } \\
\text { las compañías qué tienen que hacer. } \\
\text { La regulación limita el negocio y } \\
\text { crea desempleo. La auto-regulación } \\
\text { funciona mejor y es más barata para } \\
\text { los contribuyentes }\end{array}$ & $\begin{array}{l}\text { Los problemas de salud y medioambientales generados } \\
\text { por el modelo de negocio basado en el hiperconsumo } \\
\text { demuestran que la auto-regulación no funciona; la } \\
\text { externalización de los costes de las consecuencias del } \\
\text { modelo económico (gastos sanitarios y medioambienta- } \\
\text { les) a los contribuyentes y familias, sale finalmente más } \\
\text { caro que implementar controles y no evita el daño }\end{array}$ \\
\hline $\begin{array}{l}\text { El libre comercio es bueno para } \\
\text { todos }\end{array}$ & $\begin{array}{l}\text { El modelo económico actual no está basado en el } \\
\text { libremercado sino que está hiper-regulado a favor de } \\
\text { los intereses de los oligopolios. El mito de que los vicios } \\
\text { privados se convierten, gracias al mercado, en virtudes } \\
\text { públicas ("la mano invisible") no se ha demostrado } \\
\text { empíricamente en una economía altamente regulada. } \\
\text { La evidencia es que los mecanismos de mercado han } \\
\text { fracasado estrepitosamente, por ejemplo, al impedir el } \\
\text { acceso a servicios médicos básicos o medicamentos } \\
\text { esenciales a una gran mayoría de la población mundial }\end{array}$ \\
\hline $\begin{array}{l}\text { El consumo es esencial para el creci- } \\
\text { miento económico y la prosperidad }\end{array}$ & $\begin{array}{l}\text { El crecimiento económico ilimitado es imposible en un } \\
\text { mundo con recursos materiales limitados y, además, no } \\
\text { se correlaciona empíricamente con mejores indicadores } \\
\text { de calidad de vida sino al contrario }\end{array}$ \\
\hline
\end{tabular}

Fuene: elaboración propia basado en Freudemberg, 2014. 
En la tabla 4 se presentan confrontados las opiniones con que se autojustifican los expertos del CCCs respecto al punto de vista de los autores de la postmedicina. Así por ejemplo, desde el CCCs la responsabilidad del estilo de vida poco saludable se le atribuye al individuo mientras que los autores de la postmedicina ven esos hábitos relacionados con determinantes socioeconómicas modificables. Otra de las ideas del CCCs es que las corporaciones fabrican los productos que la gente pide como si no se diese mayormente el caso de crear necesidades inducidas. Naturalmente el CCCs esta en contra de la intromisión del Estado en sus asuntos aseverando que el libre mercado funciona; si bien desde la postmedicina se entiende que en la medida en que el CCCs externaliza los costes, el Estado tiene una responsabilidad de impedir actuaciones que puedan repercutir en la salud de los ciudadanos.

\section{Programa postmédico de actuaciones en el ámbito de la política y de la ciudadanía}

El programa post-médico estaría constituido por una batería de actuaciones coherentes que podemos agrupar en dos ámbitos: el de las políticas y el de los ciudadanos. En las páginas que siguen se presentan los resultados del análisis de las propuestas de intervención estudiadas por autores de la postmedicina.

\subsection{Programa postmédico en el ámbito de las políticas}

En el ámbito de las políticas, desde la postmedicina encontramos que se abordan los problemas de eficiencia asignativa de los recursos, para determinar -de manera pública, participativa y transparente- tanto la sostenibilidad externa (establecimiento de prioridades en el gasto público) como la sostenibilidad interna (establecimiento de prioridades en el gasto sanitario) (Repullo et al., 2006).

En relación con la sostenibilidad externa es importante reiterar que las respuestas están condicionadas por la constatación de que más inversión en sanidad no aporta más salud (e incluso la disminuye) y que desde la postmedicina uno de los criterios es que el rendimiento, en términos de bienestar, que tendría el asignar fondos a otras políticas sociales podría ser mucho mayor (Berwick, 2014).

Todo no se puede (ni procede) pagar en medicina. La única manera de poder asignar más recursos a otras políticas no sanitarias es obteniéndolos de la disminución del gasto superfluo sanitario, proceso que se ha denominado desinversión (Repullo, 2012).

Las actuaciones consistirían en desinvertir en lo innecesario (dejar de pagar por intervenciones, medicamentos o tecnologías cuya finalidad pueda obtenerse mediante otros medios más sencillos, por ejemplo, bajar de peso para controlar la tensión arterial en vez de medicamentos). Desinvertir en lo inútil consiste igualmente en dejar de pagar intervenciones, medicamentos o tecnologías que no han demostrado su efectividad; por ejemplo, muchas terapias fisioterápicas. Desinvertir en lo ineficiente consiste en dejar de pagar más cuando existen alternativas más baratas; por ejemplo, con los medicamentos y, finalmente, en lo inclemente (dejar de pagar intervenciones, medicamentos o tecnologías para pacientes que están en una situación demasiado avanzada para beneficiarse; por ejemplo, terapias al final de la vida como la quimioterapia paliativa o tecnologías como los cuidados intensivos o la diálisis). 
Tabla 5: Estrategias políticas, normativas y profesionales

\begin{tabular}{|c|c|}
\hline $\begin{array}{l}\text { Ambito de la } \\
\text { estrategia }\end{array}$ & Descripción de las actuaciones \\
\hline \multirow{7}{*}{ Políticas } & $\begin{array}{l}\text { Introducir transparencia en las estrategias de influencia o cabildeo de la industria } \\
\text { farmacéutica }\end{array}$ \\
\hline & Trasladar las políticas de medicamentos al Ministerio de Industria. \\
\hline & $\begin{array}{l}\text { Responsabilidad penal para los altos ejecutivos de la industria farmacéutica cuando se } \\
\text { demuestre daño. }\end{array}$ \\
\hline & Multas por mala conducta de las industria farmacéutica, proporcionales a sus beneficios \\
\hline & Ley de protección a informadores internos (wistleblowers) \\
\hline & Regulación de las incompatibilidades de los cargos de la administración \\
\hline & Reformas de la protección de la propiedad intelectual para una innovación responsable \\
\hline \multirow{2}{*}{ Ensayos clínicos } & $\begin{array}{l}\text { Investigación clínica con medicamentos por instituciones independientes (aunque la } \\
\text { industria farmacéutica las financie indirectamente y sea la que proponga las moléculas) }\end{array}$ \\
\hline & $\begin{array}{l}\text { Aplicar las recomendaciones AllTrials de transparencia con los datos de los ensayos } \\
\text { clínicos }\end{array}$ \\
\hline \multirow{6}{*}{ Reguladores } & $\begin{array}{l}\text { Financiar suficientemente con capital público las agencias reguladoras y evaluadoras de } \\
\text { medicamentos y tecnologías }\end{array}$ \\
\hline & Exigir ensayos clínicos más informativos \\
\hline & Separar las funciones de evaluación de las de seguridad \\
\hline & Disminuir el énfasis en la velocidad y recuperar el de la seguridad \\
\hline & Hacer cumplir la ley con los ensayos post-comercialización \\
\hline & Rediseñar la información dirigida a los ciudadanos para que sea comprensible \\
\hline \multirow{2}{*}{$\begin{array}{l}\text { Conflictos de } \\
\text { interés }\end{array}$} & $\begin{array}{l}\text { Obligación de la industria farmacéutica de declarar todas sus relaciones comerciales } \\
\text { (semejante a la Sunshine Act de EEUU) }\end{array}$ \\
\hline & $\begin{array}{l}\text { Evitar la participación de profesionales con conflictos de interés en la elaboración de } \\
\text { Guías de Práctica Clínica, recomendaciones profesionales y comisiones clínicas }\end{array}$ \\
\hline
\end{tabular}




\begin{tabular}{|c|c|}
\hline \multirow{2}{*}{$\begin{array}{l}\text { Formación y } \\
\text { congresos }\end{array}$} & $\begin{array}{l}\text { Poner en marcha un sistema de acreditación de actividades de formación continuada } \\
\text { que garantice su independencia }\end{array}$ \\
\hline & $\begin{array}{l}\text { Cambiar el modelo de reunión científica para que se puedan organizar de manera inde- } \\
\text { pendiente de la industria farmacéutica }\end{array}$ \\
\hline \multirow{2}{*}{$\begin{array}{l}\text { Actividades de } \\
\text { marketing }\end{array}$} & $\begin{array}{l}\text { Se debe prohibir el marketing de medicamentos, incluyendo las visitas comerciales, en } \\
\text { los centros sanitarios, especialmente los docentes }\end{array}$ \\
\hline & $\begin{array}{l}\text { Se debe formar a los nuevos médicos en estrategias de publicidad de la industria } \\
\text { farmacéutica }\end{array}$ \\
\hline \multirow{4}{*}{$\begin{array}{l}\text { Revistas } \\
\text { científicas }\end{array}$} & Las revistas médicas no deben tener publicidad de la industria farmacéutica \\
\hline & Publicar ensayos clínicos en espacios gratuitos, con libre acceso y sin publicidad \\
\hline & Políticas anti ghost-writers \\
\hline & Declaración de conflictos de interés de revisores y editores \\
\hline & Las investigaciones financiadas públicamente deben publicarse en libre acceso \\
\hline \multirow{2}{*}{$\begin{array}{l}\text { Medios de } \\
\text { comunicación }\end{array}$} & $\begin{array}{l}\text { Las campañas públicas de concienciación de enfermedad deben ser realizadas por } \\
\text { organismos públicos (aunque cuenten con la financiación indirecta de la industria } \\
\text { farmacéutica) }\end{array}$ \\
\hline & Declaración de conflictos de interés de periodistas \\
\hline \multirow{3}{*}{ Profesionales } & $\begin{array}{l}\text { Evitar contacto directo con la industria farmacéutica y transparencia en las } \\
\text { organizaciones }\end{array}$ \\
\hline & Declaración a los pacientes de los posibles conflictos de interés \\
\hline & $\begin{array}{l}\text { Profundizar en los valores del profesionalismo: independencia, conocimiento valido y } \\
\text { compromiso con los pacientes }\end{array}$ \\
\hline Pacientes & $\begin{array}{l}\text { Evitar la financiación directa de las asociaciones de pacientes por la industria } \\
\text { farmacéutica }\end{array}$ \\
\hline
\end{tabular}

Fuente: adaptado de Novoa, Gérvas y Ponte, 2014.

En relación con la sostenibilidad interna, autores como Callahan (2000) propugnan cambiar: las actuales prioridades por la atención hospitalaria y altamente tecnologizada, por la atención primaria y la salud pública; la curación y prolongación de la vida a toda costa por la paliación, el cuidado y la aceptación de los límites de la vida humana; la búsqueda prioritaria del beneficio individual y la infinita mejora de la calidad por el énfasis en la evaluación poblacional del impacto de las intervenciones. En palabras de Callahan (2000), "un cuidado de la salud equitativo siempre requiere sacrificios por parte de los pacientes individuales" (D.Callahan, 2000: 96).

Estar en la parte plana de la curva de rendimientos de la inversión en sanidad en absoluto significa renunciar a las posibles ventajas de tratamientos y tecnologías que puedan mejorar la salud. Se trata de ser más inteligentes para conseguirlo. Forta- 
lecer la base científica de las decisiones de crecimiento de la oferta sanitaria (evaluación de tecnologías e investigación de servicios sanitarios) introduce elementos moduladores a favor de la efectividad en la innovación (Repullo et al., 2006).

Los profesionales juegan un papel fundamental en la sostenibilidad interna ya que, a diferencia de otros empleados públicos, son asignadores finalistas de recursos, es decir, determinan sin limitaciones quién recibe qué procedimientos o servicios, cuándo y con qué intensidad. La propia naturaleza de las decisiones clínicas está dominada por elementos como la incertidumbre, la información limitada o la interacción con el paciente, lo que introduce una inevitable subjetividad en la evaluación de las situaciones y la toma de decisiones.

En la tabla 6 se sintetizan recomendaciones de actuación contra las malas prácticas corporativas

\title{
Tabla 6: Marco de actuación contra las prácticas corporativas causantes de mala salud
}

\author{
En una democracia moderna, los pre-requisitos para la salud -aire limpio, comida saludable, productos de \\ consumo seguros- deberían ser derechos y no privilegios
} Las políticas públicas deberían fomentar que las decisiones saludables individuales fueran las más sencillas,
impidiendo beneficios privados a expensa de la salud de la población

Una sociedad decente debe proteger a los niños y a otras poblaciones vulnerables del especial interés de las corporaciones por instar a comportamientos y estilos de vida poco saludables

La primera misión del gobierno es proteger la salud pública. Solamente el gobierno tiene los recursos y la posición para llevar a cabo este mandato eficazmente

La disminución del poder del gobierno no siempre incrementa proporcionalmente el de las personas sino que se cede poder a las corporaciones

La protección y la expansión de nuestros derechos democráticos otorga a las personas más oportunidades para mejorar las decisiones que afectan a sus vidas; disminuir esos derechos, otorga ese poder a otros, especialmente las grandes corporaciones

Fuente: adaptado de Feudemberg, 2014

Para Emmanual y Fuchs (19989 la variable que más influye en lo que se ha denominado la "tormenta perfecta" de la sobreutilización -además de la educación médica, la medicina defensiva o las demandas de los ciudadanos- es la influencia del mercado en las decisiones médicas. Se han propuesto algunas soluciones como las recogidas por Novoa, Gérvas y Ponte (2014). (véase Tabla 5)

En programa de políticas de la postmedicina destacan la búsqueda de estrategias de salud pública de tipo político y regulador para contrarestar la actividad del CCCS, estableciendo objetivos orientados a obtener un marco de actuación presidido por virtudes democráticas como la transparencia, la rendición de cuentas, la participación y la elección individual informada y libre (véanse Tablas 6 y 7)

Tabla 7: Una agenda política para el movimiento por un futuro más saludable

Expandir el derecho de los consumidores a saber y el deber de las corporaciones a considerar los efectos perniciosos de sus prácticas y productos

Exigir el pago de las compañías por los daños para la salud y el medio ambiente que ocasionan sus prácticas y productos (por ejemplo mediante un incremento de la fiscalidad)

Establecer estándares globales para productos y marketing 
Restablecer la propiedad pública de la ciencia y la tecnología

Restablecer la mano visible del gobierno en la protección de la salud pública

Controlar la capacidad de las corporaciones para influir y manipular los procesos democráticos

Fuente: adaptado de Feudemberg, 2014

\subsection{El programa postmédico en el ámbito de los ciudadanos}

En relación a los ciudadanos, el programa postmédico, impulsa su participación real tanto en la organización y evaluación del sistema de salud como en sus estrategias. También supone la necesidad de una reflexión de base comunitaria acerca de los valores que deben orientar las actuaciones del sistema, sus objetivos, el papel activo que deben jugar los ciudadanos en el cuidado de su salud y, en definitiva, una deliberación pública sobre los límites racionales de la medicina. Se trata de que la medicina pública se ponga al servicio de los ciudadanos pero no exactamente de sus deseos como consumidores, cosa bien distinta. Callahan (1987) lo expresa esta idea con las siguientes palabras:

"La indiferencia que ha mostrado la medicina para establecer una separación clara entre las necesidades sanitarias y los deseos, y también el relativismo operante acerca de qué bienes cabe esperar adecuadamente en nombre de la salud, se ponen de manifiesto al plantearse cuestiones acerca de los fines de la medicina" (Callahan, 1987: 74)

Lo cierto es que en una sociedad que privilegia lo individual, ha confinado al ámbito privado o religioso, las cuestiones importantes sobre los límites, la finitud y los objetivos adecuados de la vida humana en relación con la salud. Por eso Callahan (1987) observa la necesidad de "una concepción pública sobre la naturaleza de una buena vida" para evitar la construcción de fantasías y falsas expectativas.

"esta neutralidad de nuestras atomizadas y liberales sociedades modernas, es solo aparente; esconde, debido a la presión de la publicidad, los modelos de vida hollywoodiense, el continuo avance tecnológico, los intereses del mercado, etc, una coacción de fondo que podría forzar a los ciudadanos a la búsqueda insana de fantasías como la vida eterna o la posibilidad de que desaparezcan las limitaciones por las enfermedades o el dolor" (Callahan, 1987: 43).

Por eso, al igual que la utilización del coche, el reciclaje de los desechos, el consumo de productos derivados de las ballenas, etc... se han convertido en cuestiones públicas -por más que sean conductas individuales. El programa post-médico también reivindica que asuntos como el sentido del envejecimiento o los valores en relación con la salud y la enfermedad sean abordados como cuestiones de deliberación pública considerando sus implicaciones sociales. Callahan (1987) propugna que le busquemos el sentido colectivamente:

"Lo importante al respecto no es la consecución de la certeza sino la comprensión de que las cuestiones relativas al sentido y al significado son fecundas y merece la pena que busquemos conjuntamente dicho sentido y significado como comunidad, y no únicamente en nuestros ensueños y preocupaciones privadas" (Callahan, 1987: 45)

En este paso de lo individual a lo público, lo que hasta ahora se ha visualizado como un problema personal -como tener asma, estar obeso, sufrir diabetes, tener un accidentes de tráfico o sufrir un desahucio por el impago de la hipotecas- podría 
comenzar a ser visto por los ciudadanos como un probema social, consecuencia de un modelo económico al servicio de los intereses del CCCS y del fracaso de las políticas públicas (Freudemberg, 2014). Dicho de otra manera, cuando organizaciones de pacientes con SIDA, padres de niños asmáticos o asociaciones de consumidores, consiguan conectar experiencias personales con el análisis político de sus causas últimas, entónces, se conseguirá movilizar a la sociedad (la Plataforma de Afectados por las Hipotecas es un excelente ejemplo de ello).

La transformación, de problemas individuales que condicionan mala salud, en retos políticos de naturaleza pública, suele generar en la sociedad un reconocimiento de que la acción colectiva es una parte fundamental en la búsqueda de soluciones (Brown, Zavetosky, McCormick, Mayer, Morello-Frosh, Gasior, 2004).

\section{a) La simplicidad voluntaria aplicada a la sanidad y la participación comunitaria}

Serge Latouche (2008) maneja el término o concepto de "simplicidad voluntaria" entendida como reducción del consumo, conciliación de vida laboral y social, movilidad activa, preponderancia de lo local sobre lo global, sostenibilidad rural, trabajo compartido, recuperación del ocio productivo, solidaridad y cooperación, cuidado y defensa activa del medio ambiente.

Aplicando la "simplicidad voluntaria" a la sanidad le habríamos de añadir: la necesidad de deliberar públicamente y generar una concepción equilibrada y realista de los objetivos de la atención sanitaria y de los límites de la medicina; recuperar la soberanía personal sobre la propia salud; impulsar la participación activa de los ciudadanos en la organización, planificación y evaluación del sistema sanitario así como el fomento de la participación en actividades comunitarias autogestionadas generadoras de salud o salutogénesis (Burns, 2014).

Callahan (1987) ya fue pionero en plantear la simplicidad, si bien él la denominaba "setting limits" (establecer límites). Este autor enumera las virtudes que podrían ejemplificar la simplicidad voluntaria aplicada al ámbito de la salud y el envejecimiento, con las siguientes palabras:

"El coraje frente a cierta decadencia y la muerte; la humildad en respuesta al deterioro progresivo y la humillación del cuerpo, y la dignidad que puede llevar consigo; la paciencia que surge de la necesidad de gobernarse a uno mismo cuando la pérdida de control comienza a ganar terreno; la simplicidad como forma de viajar ligero de equipaje; la benignidad para evitar la tendencia a la avaricia, al afán de posesión y a la manipulación y, sorprendentemente, la hilaridad, una alegría que aparece en aquéllos que han visto mucho, hecho mucho y lamentado mucho, y que ahora pueden volar por encima del escenario humano, contemplándolo" (CaIlahan 1987: 65)

\section{b) Enfoque hacia mayor justicia}

Por último, otros autores como Sen (2000) y Nussbaum (2012), reflexionan sobre reenfocar las políticas públicas hacia una mayor justicia, generando las condiciones necesarias para que los ciudadanos desarrollen sus capacidades y sean agentes activos del cambio y no meros receptores pasivos de prestaciones (véaseTabla 8) 
Tabla 8: Diez capacidades centrales que un orden político aceptable está obligado a procurar a todos los ciudadanos

\begin{tabular}{|c|c|}
\hline $\begin{array}{l}\text { Ambito de la } \\
\text { capacidad }\end{array}$ & Descripción del poder que lleva implícito el disfrute de la capacidad \\
\hline Vida & $\begin{array}{l}\text { Poder vivir hasta el término de una vida humana de una duración normal; } \\
\text { no morir de forma prematura o antes de que la propia vida se vea tan } \\
\text { reducida que no merezca la pena vivirla }\end{array}$ \\
\hline Salud física & $\begin{array}{l}\text { Poder mantener una buena salud, incluida la salud reproductiva; recibir } \\
\text { una alimentación adecuada; disponer de un lugar apropiado para vivir }\end{array}$ \\
\hline Integridad física & $\begin{array}{l}\text { Poder desplazarse libremente de un lugar a otro; estar protegidos de } \\
\text { los ataques violentos, incluidas las agresiones sexuales y la violencia } \\
\text { doméstica }\end{array}$ \\
\hline $\begin{array}{l}\text { Sentidos, imagina- } \\
\text { ción y pensamiento }\end{array}$ & $\begin{array}{l}\text { Poder utilizarlos... y hacerlo de un modo formado y cultivado por una } \\
\text { educación adecuada }\end{array}$ \\
\hline Emociones & $\begin{array}{l}\text { Poder amar, apenarse, sentir añoranza, gratitud e indignación justificada. } \\
\text { Que no se malogre nuestro desarrollo emocional por culpa del miedo y la } \\
\text { ansiedad }\end{array}$ \\
\hline Razón práctica & $\begin{array}{l}\text { Poder formarse una concepción del bien y reflexionar críticamente } \\
\text { acerca de la planificación de la propia vida (protección de la libertad de } \\
\text { conciencia y de observancia religiosa) }\end{array}$ \\
\hline Afiliación & $\begin{array}{l}\text { Participar en formas diversas de interacción social; disponer de las bases } \\
\text { sociales necesarias para que no sintamos humillación y sí respeto por } \\
\text { nosotros mismos }\end{array}$ \\
\hline Otras especies & $\begin{array}{l}\text { Poder vivir una relación próxima y respetuosa con los animales, las } \\
\text { plantas y el mundo natural }\end{array}$ \\
\hline Juego & Poder reír jugar y disfrutar de actividades recreativas \\
\hline $\begin{array}{l}\text { Control sobre el } \\
\text { propio entorno }\end{array}$ & $\begin{array}{l}\text { Poder participar de forma efectiva en las decisiones políticas que gobier- } \\
\text { nan nuestra vida; posee propiedades y ostentar derechos de propiedad } \\
\text { en igualdad de condiciones con las demás personas; tener derecho a } \\
\text { buscar trabajo en un plano de igualdad con los demás }\end{array}$ \\
\hline
\end{tabular}

Fuente: adaptado de Nussbaum, 2012

Como se desprende del cuadro 8, el disfrute de las 10 capacidades enunciadas por Nussbaum (2012), en parte están recogidos en la declaración de derechos humanos, en el índice de desarrollo humano y en la definición de salud de la OMS. Su disfrute va mas allá de la salud física individual pero también inciden en ella y son una muestra de cómo hay un cúmulo de actuaciones y políticas no sanitarias que están siendo descuidadas cuando pueden tener una repercusión importante en aumentar los niveles de salud y bienestar general.

\section{Conclusiones}

La desproporción entre los recursos invertidos en el sistema sanitario público y los resultados obtenidos expresan una llamada de atención hacia la necesidad de reflexión y cambios. Ya que además está suponiendo una lacra financiera para desarrollar otras políticas públicas. 
Hemos denominado post-medicina al movimiento, de base eco-política, que aboga por superar los principios rectores (implícitos y explícitos) de un sistema médico que ha perdido la visión de sus fines (Hasting, 1996) y está dominado por el Complejo Corporativo Consumista Sanitario cuya actividad repercute directamente e indirectamente sobre la salud.

El programa post-médico propone decrecer en atención sanitaria para poder crecer en salud y equidad.

La crisis de la sanidad sería estructural al compartir con las crisis financiera, social y ecológica el nexo de haberse guiado por un modelo económico y social de hiperconsumo; un desarrollo tecnológico guiado por las necesidades impuestas por el mercado a través de la publicidad; el individualismo hedonista que carece de una visión compartida de sociedad, o la progresiva degradación democrática que permite que los intereses particulares (económicos y otros) prevalezcan sobre los comunes.

De tal manera que la sanidad pública se habría convertido en un gigantesco mercado subvencionado, con un gasto cada vez menos rentable socialmente porque, entre otras cosas, la biomedicina adoptó fines irracionales impuestos por el mercado y tácitamente pasó a equipara el derecho a la salud con la exigencia social a no tener sufrimiento, enfermedades o riesgos. Además de la explosión del gasto público sanitario, esto conlleva graves consecuencias adicionales, al impedir la financiación pública suficiente de otras políticas con capacidad de generar salud y equidad

El desarrollismo ilimitado, del que la atención sanitaria es una parte muy importante, podrá remitir como modelo dominante de los sistemas públicos de salud. La crisis del paradigma biomédico nos pone delante de un enorme fracaso social. No es la metáfora de la abundancia la que dará respuestas a los retos a los que nos enfrentamos sino la de la escasez y la contención. La dificultad estriba en que a la pulsión expansionista de la actividad del CCCS y su cobertura ideológica se le suma una falta de debate público sobre las características del problema y sus soluciones alternativas. Esto nos lleva a una situación actual de escasa capacidad de reacción política, social y profesional.

El Complejo Corporativo Consumista Sanitario (CCCS), que es una red de compañías que actúan como oligopolios mundiales, se ha convertido en la principal causa evitable de morbimortalidad en el mundo. La salud pública, por tanto, debe enfocar sus esfuerzos a estrategias preventivas más de índole política, regulatoria y sociocultural que a las tradicionales de educación para la salud o prevención. El modelo de negocio del CCCS dirigido por la publicidad, ha generado un contexto de insuficiencia de las salvaguardas tradicionales de la medicina -institucionales, científicas y profesionales-, que ha puesto en peligro la seguridad de los pacientes, el prestigio de la ciencia y la medicina, la sostenibilidad de los sistemas de salud, la equidad y la propia viabilidad de las industrias farmacéuticas en el largo plazo.

Las propuestas de intervención que proponen los autores de la postmedicina los clasificamos en de ámbito político y de ámbito ciudadano. Las políticas se refieren a la administración eficiente, participativas y transparente. Es lo que Repullo (2012) denomina la desinversion en lo innecesario, lo inútil y lo ineficiente. En este mismo ámbito se clasifican las propuestas legislativas para evitar que se desvíen fondos al servicio de intereses privados y que se incluyen otras medidas como las políticas de medicamentos, datos de ensayos clínicos, control del marketing de medicamentos, el libre acceso a las publicaciones científicas, etc. . Por lo que a la sostenibilidad interna se refiere, las propuestas van en la línea de fortalecer la base científica de 
las decisiones, restablecer estándares globales para los productos o garantizar el derecho de los consumidores a saber sobre los efectos perniciosos de los productos de consumo.

En cuanto a las propuestas de intervención en el ámbito ciudadano, van mayormente dirigidas a implicar mas a la población en la información sobre el sistema médico y sus mejoras de funcionamiento. Destaca la propuesta de hacer partícipe a la ciudadania de la deliberacion pública sobre los límites de la medicina y de la deriva que ha tomado una sanidad que se puso al servicio de los deseos de los consumidores olvidando su función de dar servicio a los ciudadanos.

\section{Bibliografía}

Berwick DM; Andrew D. \& Hackbarth M (2012). Eliminating Waste in US Health Care. The Journal of the American Medical Association (JAMA). 2012; 307(14):1513-1516. doi. org/10.1001/jama.2012.362

Berwick, Donald M. (2014). Reshaping US Health Care. From Competition and Confiscation to Cooperation and Mobilization. The Journal of the American Medical Association (JAMA) 2014;312(20):2099-2100 doi:10.1001/jama.2014.15727.

Burns, H (2014). What causes health? Editorial J R Coll Physicians Edinb 2014; 44; 103-5 doi.org/10.4997/JRCPE.2014.202

Brown P; Zavetosky S; McCormick S; Mayer B; Morello-Frosh R \& Gasior R. (2004). Embodied health movements: new approaches to social movements. Health. Sociology of Health \& Illness. 2004;26(1):50-80. doi.org/10.1111/j.1467-9566.2004.00378.x

Callahan, Daniel (1987) Setting Limits: Medical Goals in an Aging Society. New York: Simon and Schuster. Traducido en Callahan, Daniel (2004): Poner límites. Los fines de la medicina en una sociedad que envejece. Madrid: Editorial Triacastela.

Callahan, Daniel (1998). False hopes: overcoming the obstacles to a sustainable, affordable medicine. Nueva York: Ed.Simon \& Shuster

Callahan, Daniel (2000). La inevitable tensión entre la igualdad, la calidad y los derechos de los pacientes. En Lolas-Stepke, Fernando (Editor). Bioética y cuidado de la salud: Equidad, Calidad, Derechos. Programa Regional de Bioética (OPS) /Organización Mundial de la Salud. .Panamá, 2000.

Cortés-Franch I \& González B (2014) Crisis económico-financiera y salud en España. Evidencia y perpectiva. Informe SESPAS 2014 Gac Sanit.2014;28 Supl 1:1-6 - Vol. 28.

Emmanuel,EJ \& Fuchs, VR (2008). The perfect storm of overutilization Journal of American Medical Association JAMA. 2008 Jun 18;299(23):2789-91. doi.org/10.1001/ jama.299.23.2789

Freudemberg, Nicholas (2014). Lethal but legal: corporations, consumption and protecting public health. Oxford: Oxford University Press

Fuchs, VR (2004). More variation in use of care, more flat-of-the-curve medicine. Health affairs; Suppl Variation; VAR104-7 doi.org/10.1377/hlthaff.var.104

Gérvas J y Pérez-Fernández M (2013) Sano y salvo (y libre de intervenciones médicas innecesarias). Ed. Los libros del lince. Barcelona. 
Gérvas J y Pérez-Fernández M (2014) Sobrediagnóstico, un problema clinico, ético y social FMC. 2014;21(3):137-42

Griffiths J \& col (Eds) (2009). The Health Practitioner's Guide to Climate Change: Diagnosis and Cure. London: Ed. Earthscan.

Gøtzsche P. (2014). Medicamentos que matan y crimen organizado: Cómo las grandes farmacéuticas han corrompido el sistema de salud. Ed. Libros del Lince, Barcelona.

Hasting Center Report (1996). The Goals of Medicine: Setting New Priorities, Hastings Center Report 26,6 (disponible en castellano en la web bioeticanet),

Healy D (2012). Pharmaggedon. Berkeley: University of California Press.

Hernández I, Lumbreras B. (2014) Crisis e independencia de las políticas de salud pública SESPAS 2014 Gac Sanit.2014;28 Supl 1:24-30 - Vol. 28.

Judt T (2010). Algo va mal. Madrid: Taurus.

Kohn LT; Carrigan JM; Donaldson MS (Ed.) (......) To Err Is Human: Building a Safer Health System. Institute of Medicine. National Academy Press: Washington

Latouche S (2008). La apuesta por el decrecimiento, Barcelona: Ed.Icaria

Marmot M (2004). Status Syndrome: How your Social Standing Directly Affects Your Health and Life Expectancy. London: Ed. Bloomsbury.

McMichael A (2013). Globalization, Climate Change and Human Health. N Engl J Med 2013;368:1335-43. DOI: 10.1056/NEJMra1109341. doi.org/10.1056/NEJMra1109341 . (Versión traducida "Globalización, cambio climático y salud humana" en 04/04/2013 www.nogracias.

Novoa A. (2013a). No podemos esperar y cruzar los dedos. Aten Primaria (Editorial) 2013; 45(1): 1-2.

Novoa A. (2013b). Postmedicina: decrecer en atención sanitaria para crecer en salud y equidad. En FJ Lopez Frías y col (Eds) "Bioética, neuroética, libertad y justicia" Granada: Editorial Comares (pag 303-334).

Novoa A; Gérvas J y Ponte C. (2014). Salvaguardas, deriva institucional e industrias farmacéuticas. AMF 2014;10(7):373-382 doi.org/10.1016/j.aprim.2012.12.001.

Nussbaum M. (2012). Crear capacidades: propuesta para el desarrollo humano. BarceIona: Ed. Paidós

OMS (2008). Informe sobre la salud en el mundo. La atención primaria de salud: Más importante que nunca. Who Publications.

OMS (2009). Informe final de la Comisión sobre Determinantes Sociales de la Salud: "Subsanar las desigualdades en una generación: alcanzar la equidad sanitaria actuando sobre los determinantes sociales de la salud". Who Publications.

Peiró, Salvador (2012). Para salir del hoyo, lo primero es dejar de cavar. Atención Primaria, Volume 44, Issue 12, Pages 691-694

Peiró S; Meneu R y Bernal E (1999). Tres tristes tópicos sobre las variaciones en la práctica médica. Gestión Clínica y Sanitaria, Editorial del vol 7; n² 2. Ed. Fundación IISS, Valencia, 1999. 
Repullo JR y Segura A (2006) Salud pública y sostenibilidad de los sistemas públicos de salud. Revista Española de Salud Pública 2006; 80: 475-482. doi.org/10.1590/ S1135-57272006000500006

Repullo JR (2012). Taxonomía práctica de la «desinversión sanitaria» en lo que no añade valor, para hacer sostenible el Sistema Nacional de Salud. Rev Cal Asist, 2012; 27:130-8. - vol.27, n.3.

Segura A (2014). Recortes, austeridad y salud. Informe SESPAS 2014. Gaceta Sanitaria. 2014;28 Supl 1:7-11 doi.org/10.1016/j.gaceta.2014.02.009

Sen, AK (2000). Desarrollo y libertad. Barcelona: Editorial Planeta.

Stamatakis E; Weiler R; loannidis J. (2014). Undue industry influences that distort healthcare research, strategy, expenditure and practice: a review. Eur J Clin Invest. 2013 May;43(5):469-75.

Taibo C (2009). En defensa del decrecimiento: sobre capitalismo, crisis y barbarie. Madrid: Ed. Los libros de la catarata. 
